\title{
On generalizations of the Jacobsthal sequence
}

\author{
Fügen Torunbalcı Aydın \\ Yildiz Technical University \\ Faculty of Chemical and Metallurgical Engineering \\ Department of Mathematical Engineering \\ Davutpasa Campus, 34220, Esenler, Istanbul, Turkey \\ e-mails: faydin@yildiz.edu.tr, ftorunay@gmail.com
}

Received: 1 December 2017 Revised: 13 February $2018 \quad$ Accepted: 16 February 2018

\begin{abstract}
In this paper, the generalized Jacobsthal and generalized complex Jacobsthal and generalized dual Jacobsthal sequences using the Jacobsthal numbers are investigated. Also, special cases of these sequences are investigated. Furthermore, recurrence relations, vectors, the golden ratio and Binet's formula for the generalized Jacobsthal sequences and generalized dual Jacobsthal sequences are given.
\end{abstract}

Keywords: Jacobsthal number, Jacobsthal-Lucas number, Jacobsthal sequence, Generalized Jacobsthal sequence, Generalized complex Jacobsthal sequence, Generalized dual Jacobsthal sequence.

2010 Mathematics Subject Classification: 11B37, 11B50, 11R52.

\section{Introduction}

In 1973, the first use of these numbers appears "A Handbook of Integer Sequences" in a paper by Sloane by the title applications of Jacobsthal sequences to curves [17].

Further, in 1988, Horadam [12] introduced the Jacobsthal and Jacobsthal-Lucas sequences recurrence relation $\left\{J_{n}\right\}$ and $\left\{j_{n}\right\}$ are defined by the recurrence relations

$$
\begin{gathered}
J_{0}=0, \quad J_{1}=1, \quad J_{n}=J_{n-1}+2 J_{n-2}, \quad \text { for } n \geq 2, \\
j_{0}=2, \quad j_{1}=1, \quad j_{n}=j_{n-1}+2 j_{n-2}, \quad \text { for } n \geq 2,
\end{gathered}
$$

respectively. 
The first eleven terms of the Jacobsthal sequence $J_{n}$ are $0,1,1,3,5,11,21,43,85$, 171 , and 341 . This sequence is given by the formula

$$
J_{n}=\frac{2^{n}-(-1)^{n}}{3} .
$$

The first eleven terms of the Jacobsthal-Lucas sequence $\left\{j_{n}\right\}$ are $2,1,5,7,17,31,65,127$, 257,511 and 1025 . This sequence is given by the formula

$$
j_{n}=2^{n}+(-1)^{n}
$$

There are many articles in the literature that study on the Jacobsthal sequences $[2,3,11,13$, 14]. Generalization of the Jacobsthal sequences is given in $[4,5,8,9,10]$. Also, we can see the matrix representations of Jacobsthal and Jacobsthal-Lucas numbers in $[6,7,15,16]$. Several authors worked on the Jacobsthal quaternions in $[1,18,19]$.

For the Jacobsthal and Jacobsthal-Lucas numbers, the following properties

$$
\begin{gathered}
J_{n}^{2}+2 J_{n-1}^{2}=J_{2 n-1} \\
J_{n+1}^{2}+2 J_{n}^{2}=J_{2 n+1} \\
J_{n+1}^{2}-4 J_{n-1}^{2}=J_{2 n} \\
J_{n}^{2}-4 J_{n-1}^{2}=(-1)^{n+1} J_{n+1} \\
J_{n-1} J_{n+1}-J_{n}^{2}=(-1)^{n} 2^{n-1} \\
J_{n+1}-4 J_{n-1}=(-1)^{n+1} \\
J_{n+1}+4 J_{n}=j_{n+1} \\
j_{n} J_{n}=J_{2 n} \\
J_{m} J_{n+1}+2 J_{m-1} J_{n}=J_{m+n} \\
J_{m} J_{n-1}-J_{m-1} J_{n}=(-1)^{n} 2^{n-1} J_{m-n}
\end{gathered}
$$

hold $[6,7,13,14,15,16,18,19]$.

\section{The generalized Jacobsthal sequence}

In this section, the generalized Jacobsthal sequence denoted by $\mathbb{J}_{n}$ will be defined. The generalized Jacobsthal sequence is defined by

$$
\mathbb{J}_{n}=\mathbb{J}_{n-1}+2 \mathbb{J}_{n-2}, \quad(n \geq 3)
$$

with $\mathbb{J}_{0}=q, \mathbb{J}_{1}=p+q, \mathbb{J}_{2}=p+3 q$, where $p, q$ are arbitrary integers. That is, the generalized Jacobsthal sequence is

$$
q, p+q, p+3 q, 3 p+5 q, 5 p+11 q, 11 p+21 q, \ldots,(p+q) J_{n}+2 q J_{n-1}, \ldots
$$


Using the equations (11) and (12), we get

$$
\begin{aligned}
\mathbb{J}_{n} & =p J_{n}+q J_{n+1} \\
\mathbb{J}_{n+1} & =(p+q) J_{n+1}+2 q J_{n} \\
\mathbb{J}_{n+2} & =(p+3 q) J_{n+1}+2(p+q) J_{n} .
\end{aligned}
$$

Putting $n=r$ in (13) and using (11), we find

$$
\begin{aligned}
& \mathbb{J}_{r+3}=(3 p+5 q) J_{r+1}+2(p+3 q) J_{r}=\mathbb{J}_{3} J_{r+1}+2 \mathbb{J}_{2} J_{r} \\
& \mathbb{J}_{r+4}=(5 p+11 q) J_{r+1}+2(3 p+5 q) J_{r}=\mathbb{J}_{4} J_{r+1}+2 \mathbb{J}_{3} J_{r} .
\end{aligned}
$$

So, in general, we obtain relation between generalized Jacobsthal sequence and Jacobsthal sequence as follows:

$$
\mathbb{J}_{n+r}=\mathbb{J}_{n} J_{r+1}+2 \mathbb{J}_{n-1} J_{r} .
$$

Also, certain results follow almost immediately from (11)

$$
\mathbb{J}_{n+2}-3 \mathbb{J}_{n}-2 \mathbb{J}_{n-1}=0 .
$$

For the generalized Jacobsthal sequence, we have the following properties:

$$
\begin{gathered}
\left(\mathbb{J}_{n}\right)^{2}+2\left(\mathbb{J}_{n-1}\right)^{2}=(2 p+q) \mathbb{J}_{2 n-1}-e_{J} J_{2 n-1}, \\
\left(\mathbb{J}_{n+1}\right)^{2}-4\left(\mathbb{J}_{n-1}\right)^{2}=(2 p+q) \mathbb{J}_{2 n}-e_{J} J_{2 n}, \\
\mathbb{J}_{n-1} \mathbb{J}_{n+1}-\left(\mathbb{J}_{n}\right)^{2}=(-1)^{n} 2^{n-1} e_{J}, \\
\mathbb{J}_{n-r} \mathbb{J}_{n+r}-\left(\mathbb{J}_{n}\right)^{2}=(-1)^{n-r+1} 2^{n-r} J_{r}^{2} e_{J}, \\
4\left(\mathbb{J}_{n}\right)^{2}+2 e_{J} J_{n+1}^{2}=2 p \mathbb{J}_{2 n+1}, \\
\mathbb{J}_{m} \mathbb{J}_{n+1}+2 \mathbb{J}_{m-1} \mathbb{J}_{n}=(2 p+q) \mathbb{J}_{m+n}-e_{J} J_{m+n}, \\
\mathbb{J}_{m} \mathbb{J}_{n-1}-\mathbb{J}_{m-1} \mathbb{J}_{n}=(-1)^{n} 2^{n-1} e_{J} J_{m-n},
\end{gathered}
$$

where $e_{J}=p^{2}+p q-2 q^{2}$.

Also, for $p=1, q=0$, we get the well-known results in $(1-10)$.

Theorem 1. If $\mathbb{J}_{n}$ is the generalized Jacobsthal number, then

$$
\lim _{n \rightarrow \infty} \frac{\mathbb{J}_{n+1}}{\mathbb{J}_{n}}=\frac{(p+q) \alpha+2 q}{q \alpha+p}
$$

where $\alpha=2$.

Proof. We have for the Jacobsthal number $J_{n}$,

$$
\lim _{n \rightarrow \infty} \frac{J_{n+1}}{J_{n}}=\alpha,
$$

where $\alpha=2$ [17].

Then for the generalized Jacobsthal number $\mathbb{J}_{n}$, we obtain

$$
\lim _{n \rightarrow \infty} \frac{\mathbb{J}_{n+1}}{\mathbb{J}_{n}}=\lim _{n \rightarrow \infty} \frac{(p+q) J_{n+1}+2 q J_{n}}{p J_{n}+q J_{n+1}}=\frac{(p+q) \alpha+2 q}{q \alpha+p} .
$$


Theorem 2. The Binet's formula ${ }^{1}$ for the generalized Jacobsthal sequence is as follows;

$$
\mathbb{J}_{n}=\frac{\left(\bar{\alpha} \alpha^{n}-\bar{\beta} \beta^{n}\right)}{\alpha-\beta} .
$$

Proof. The characteristic equation of recurrence relation $\mathbb{J}_{n+2}=\mathbb{J}_{n+1}+2 \mathbb{J}_{n}$ is

$$
t^{2}-t-2=0
$$

The roots of this equation are

$$
\alpha=-1 \text { and } \beta=2,
$$

where $\alpha+\beta=1, \quad \alpha-\beta=3, \alpha \beta=-2$. Using recurrence relation and initial values $\mathbb{J}_{0}=q$, $\mathbb{J}_{1}=p+q$, for the Binet's formula $\mathbb{J}_{n}$, we get

$$
\mathbb{J}_{n}=A \alpha^{n}+B \beta^{n}=\frac{\left(\bar{\alpha} \alpha^{n}-\bar{\beta} \beta^{n}\right)}{\alpha-\beta},
$$

where $A=\frac{\mathbb{J}_{1}-\beta \mathbb{J}_{0}}{\alpha-\beta}, B=\frac{\alpha \mathbb{J}_{0}-\mathbb{J}_{1}}{\alpha-\beta}$ and $\bar{\alpha}=p+q(1-\beta), \bar{\beta}=q(\alpha-1)-p$.

\subsection{The generalized Jacobsthal vectors}

A generalized Jacobsthal vector is defined by $\overrightarrow{\mathbb{J}}_{\mathrm{n}}=\left(\mathbb{J}_{n}, \mathbb{J}_{n+1}, \mathbb{J}_{n+2}\right)$. Also, from equation (12) it can be expressed as

$$
{\overrightarrow{\mathbb{J}_{\mathrm{n}}}}=(p+q) \overrightarrow{\mathrm{J}}_{n}+2 q \overrightarrow{\mathrm{J}}_{\mathrm{n}-1},
$$

where $\vec{J}_{\mathrm{n}}=\left(J_{n}, J_{n+1}, J_{n+2}\right)$ and $\vec{J}_{\mathrm{n}+1}=\left(J_{n+1}, J_{n+2}, J_{n+3}\right)$ are the Jacobsthal vectors. The product of $\overrightarrow{\mathbb{J}_{\mathrm{n}}}$ and $\lambda \in \mathbb{R}$ is given by

$$
\lambda \overrightarrow{\mathbb{J}}_{\mathrm{n}}=\left(\lambda \overrightarrow{\mathbb{J}}_{\mathrm{n}}, \lambda \overrightarrow{\mathbb{J}}_{\mathrm{n}+1}, \lambda \overrightarrow{\mathbb{J}}_{\mathrm{n}+2}\right)
$$

and $\overrightarrow{\mathbb{J}_{\mathrm{n}}}$ and $\overrightarrow{\mathbb{J}_{\mathrm{m}}}$ are equal if and only if $\mathbb{J}_{n}=\mathbb{J}_{m}, \mathbb{J}_{n+1}=\mathbb{J}_{m+1}, \mathbb{J}_{n+2}=\mathbb{J}_{m+2}$.

Theorem 3. Let $\overrightarrow{\mathbb{J}}_{\mathrm{n}}$ and $\overrightarrow{\mathbb{J}}_{\mathrm{m}}$ be two generalized Jacobsthal vectors. The dot product of $\overrightarrow{\mathbb{J}}_{\mathrm{n}}$ and $\overrightarrow{\mathbb{J}}_{\mathrm{m}}$ is given by

$$
\begin{aligned}
\left\langle\overrightarrow{\mathbb{J}}_{\mathrm{n}}, \overrightarrow{\mathbb{J}}_{\mathrm{m}}\right\rangle= & p^{2}\left[\frac{1}{3}\left(J_{n+m}+J_{n+m+2}+J_{n+m+4}\right)\right. \\
& \left.+(-1)^{n+1} J_{m}+(-1)^{m+1} J_{n}\right] \\
& +2 p q\left[\frac{1}{3}\left(J_{n+m+1}+J_{n+m+3}+J_{n+m+5}\right)\right. \\
& \left.+(-1)^{n+1} J_{m-1}+(-1)^{m+1} J_{n-1}\right] \\
& +q^{2}\left[\frac{1}{3}\left(J_{n+m+2}+J_{n+m+4}+J_{n+m+6}\right)\right. \\
& \left.+(-1)^{n+2} J_{m+1}+(-1)^{m+2} J_{n+1}\right] .
\end{aligned}
$$

\footnotetext{
${ }^{1}$ Binet's formula is the explicit formula to obtain the $n$-th Jacobsthal and Jacobsthal-Lucas numbers. It is well known that for the Jacobsthal and Jacobsthal-Lucas numbers, Binet's formulas are $J_{n}=\frac{\alpha^{n}-\beta^{n}}{\alpha-\beta}$ and $j_{n}=\alpha^{n}+\beta^{n}$ respectively, where $\alpha+\beta=1, \alpha-\beta=3, \alpha \beta=-2$ and $\alpha=2, \beta=-1,[15,16]$.
} 
Proof. The dot product of $\overrightarrow{\mathbb{J}}_{\mathrm{n}}=\left(\mathbb{J}_{n}, \mathbb{J}_{n+1}, \mathbb{J}_{n+2}\right)$ and $\overrightarrow{\mathbb{J}}_{\mathrm{m}}=\left(\mathbb{J}_{m}, \mathbb{J}_{m+1}, \mathbb{J}_{m+2}\right)$ are defined by $\left\langle\overrightarrow{\mathbb{J}}_{\mathrm{n}}, \overrightarrow{\mathbb{J}}_{\mathrm{m}}\right\rangle=\mathbb{J}_{n} \mathbb{J}_{m}+\mathbb{J}_{n+1} \mathbb{J}_{m+1}+\mathbb{J}_{n+2} \mathbb{J}_{m+2}$. Also, using the equations (11), (12) and (13), we obtain

$$
\begin{aligned}
& \mathbb{J}_{n} \mathbb{J}_{m}=p^{2}\left(J_{n} J_{m}\right)+p q\left[J_{n} J_{m+1}+J_{n+1} J_{m}\right]+q^{2}\left(J_{n+1} J_{m+1}\right), \\
& \mathbb{J}_{n+1} \mathbb{J}_{m+1}= p^{2}\left(J_{n+1} J_{m+1}\right) \\
&+p q\left[J_{n+1} J_{m+2}+J_{n+2} J_{m+1}\right]+q^{2}\left(J_{n+2} J_{m+2}\right), \\
& \mathbb{J}_{n+2} \mathbb{J}_{m+2}= p^{2}\left(J_{n+2} J_{m+2}\right) \\
&+p q\left[J_{n+2} J_{m+3}+J_{n+3} J_{m+2}\right]+q^{2}\left(J_{n+3} J_{m+3}\right) .
\end{aligned}
$$

Then, from the equations (31), (32) and (33), we have

$$
\begin{aligned}
\left\langle\overrightarrow{\mathbb{J}}_{\mathrm{n}}, \overrightarrow{\mathbb{J}}_{\mathrm{m}}\right\rangle= & p^{2}\left(J_{n} J_{m}+J_{n+1} J_{m+1}+J_{n+2} J_{m+2}\right) \\
& +p q\left[J_{n} J_{m+1}+J_{n+1} J_{m}+J_{n+1} J_{m+2}\right. \\
& \left.+J_{n+2} J_{m+1}+J_{n+2} J_{m+3}+J_{n+3} J_{m+2}\right] \\
& +q^{2}\left(J_{n+1} J_{m+1}+J_{n+2} J_{m+2}+J_{n+3} J_{m+3}\right) \\
= & p^{2}\left[\frac{1}{3}\left(J_{n+m}+J_{n+m+2}+J_{n+m+4}\right)\right. \\
& \left.+(-1)^{n+1} J_{m}+(-1)^{m+1} J_{n}\right] \\
& +2 p q\left[\frac{1}{3}\left(J_{n+m+1}+J_{n+m+3}+J_{n+m+5}\right)\right. \\
& \left.+(-1)^{n+1} J_{m-1}+(-1)^{m+1} J_{n-1}\right] \\
& +q^{2}\left[\frac{1}{3}\left(J_{n+m+2}+J_{n+m+4}+J_{n+m+6}\right)\right. \\
& \left.+(-1)^{n+2} J_{m+1}+(-1)^{m+2} J_{n+1}\right] .
\end{aligned}
$$

Then for the norm of the generalized Jacobsthal vector, using identities as follows:

$$
\begin{gathered}
J_{n+1}^{2}+2 J_{n}^{2}=J_{2 n+1}, \\
J_{n+1}^{2}-J_{n}^{2}=2^{n+1} J_{n-1}, \\
J_{m} J_{n+1}+2 J_{m-1} J_{n}=J_{m+n},
\end{gathered}
$$

we have

$$
\begin{aligned}
\left\|\overrightarrow{\mathbb{J}_{\mathrm{n}}}\right\|^{2}= & \left\langle\overrightarrow{\mathbb{J}_{\mathrm{n}}}, \overrightarrow{\mathbb{J}_{\mathrm{n}}}\right\rangle=\mathbb{J}_{n}^{2}+\mathbb{J}_{n+1}^{2}+\mathbb{J}_{n+2}^{2} \\
= & p^{2}\left[\frac{1}{3}\left(J_{2 n}+J_{2 n+2}+J_{2 n+4}\right)+2(-1)^{n+1} J_{n}\right] \\
& +2 p q\left[\frac{1}{3}\left(J_{2 n+1}+J_{2 n+3}+J_{2 n+5}\right)+2(-1)^{n+1} J_{n-1}\right] \\
& +q^{2}\left[\frac{1}{3}\left(J_{2 n+2}+J_{2 n+4}+J_{2 n+6}\right)+2(-1)^{n+2} J_{n+1}\right] \\
= & p^{2}\left[J_{2 n+3}-2^{n+1} J_{n-1}\right] \\
& +2 p q\left[\frac{1}{3}\left(J_{2 n+1}+J_{2 n+3}+J_{2 n+5}\right)+2(-1)^{n+1} J_{n-1}\right] \\
& +q^{2}\left[J_{2 n+5}-2^{n+2} J_{n}\right] .
\end{aligned}
$$


- Special case 1: For the dot product of the generalized Jacobsthal vectors $\overrightarrow{\mathbb{J}}_{n}$ and $\overrightarrow{\mathbb{J}}_{n+1}$, we get

$$
\begin{aligned}
\left\langle\overrightarrow{\mathbb{J}}_{\mathrm{n}}, \overrightarrow{\mathbb{J}}_{\mathrm{n}+1}\right\rangle= & \mathbb{J}_{n} \mathbb{J}_{n+1}+\mathbb{J}_{n+1} \mathbb{J}_{n+2}+\mathbb{J}_{n+2} \mathbb{J}_{n+3} \\
= & p^{2}\left[\frac{1}{3}\left(J_{2 n+1}+J_{2 n+3}+J_{2 n+5}\right)+2(-1)^{n+1} J_{n-1}\right] \\
& +2 p q\left[\frac{1}{3}\left(J_{2 n+2}+J_{2 n+4}+J_{2 n+6}\right)+2(-1)^{n+1} J_{n-2}\right] \\
& +q^{2}\left[\frac{1}{3}\left(J_{2 n+3}+J_{2 n+5}+J_{2 n+7}\right)+2(-1)^{n} J_{n}\right]
\end{aligned}
$$

and

$$
\begin{aligned}
\left\langle\overrightarrow{\mathbb{J}}_{\mathrm{n}}, \overrightarrow{\mathbb{J}}_{\mathrm{n}}\right\rangle= & \mathbb{J}_{n}{ }^{2}+\mathbb{J}_{n+1}{ }^{2}+\mathbb{J}_{n+2}{ }^{2} \\
= & p^{2}\left[J_{n}^{2}+J_{n+1}^{2}+J_{n+2}^{2}\right] \\
& +2 p q\left[J_{n} J_{n+1}+J_{n+1} J_{n+2}+J_{n+} J_{n+3}\right] \\
& +q^{2}\left[J_{n+1}^{2}+J_{n+2}^{2}+J_{n+3}^{2}\right] \\
= & p^{2}\left[\frac{1}{3}\left(J_{2 n}+J_{2 n+2}+J_{2 n+4}\right)+2(-1)^{n+1} J_{n}\right] \\
& +2 p q\left[\frac{1}{3}\left(J_{2 n+1}+J_{2 n+3}+J_{2 n+5}\right)+(-1)^{n+1} J_{n-1}\right] \\
& +q^{2}\left[\frac{1}{3}\left(J_{2 n+2}+J_{2 n+4}+J_{2 n+6}\right)+2(-1)^{n+2} J_{n+1}\right] \\
& p^{2}\left[J_{2 n+3}-2^{n+1} J_{n-1}\right] \\
& +2 p q\left[\frac{1}{3}\left(J_{2 n+1}+J_{2 n+3}+J_{2 n+5}\right)+2(-1)^{n+1} J_{n-1}\right] \\
& +q^{2}\left[J_{2 n+5}-2^{n+2} J_{n}\right] .
\end{aligned}
$$

Theorem 4. Let $\overrightarrow{\mathbb{J}_{n}}$ and $\overrightarrow{\mathbb{J}_{m}}$ be two generalized Jacobsthal vectors. The cross product of $\overrightarrow{\mathbb{J}_{n}}$ and $\overrightarrow{\mathbb{J}_{\mathrm{m}}}$ is given by

$$
\overrightarrow{\mathbb{J}_{\mathrm{n}}} \times \overrightarrow{\mathbb{J}_{\mathrm{m}}}=(-1)^{n+1} 2^{n} J_{m-n}\left(p^{2}+p q-2 q^{2}\right)(-2 i-j+k) .
$$

Proof. The cross product of $\overrightarrow{\mathbb{J}_{\mathrm{n}}} \times \overrightarrow{\mathbb{J}_{\mathrm{m}}}$ is defined by

$$
\begin{aligned}
\overrightarrow{\mathbb{J}}_{\mathrm{n}} \times \overrightarrow{\mathbb{J}}_{\mathrm{m}}= & \left|\begin{array}{ccc}
i & j & k \\
\mathbb{J}_{n} & \mathbb{J}_{n+1} & \mathbb{J}_{n+2} \\
\mathbb{J}_{m} & \mathbb{J}_{m+1} & \mathbb{J}_{m+2}
\end{array}\right|=i\left(\mathbb{J}_{m+2} \mathbb{J}_{n+1}-\mathbb{J}_{m+1} \mathbb{J}_{n+2}\right) \\
& -j\left(\mathbb{J}_{m+2} \mathbb{J}_{n}-\mathbb{J}_{m} \mathbb{J}_{n+2}\right)+k\left(\mathbb{J}_{m+1} \mathbb{J}_{n}-\mathbb{J}_{m} \mathbb{J}_{n+1}\right) .
\end{aligned}
$$

Now, we calculate the cross products:

Using relations (11) and (13) or the property $J_{n}^{2}-J_{n+1} J_{n-1}=(-1)^{n+1} 2^{n-1}$, we get

$$
\mathbb{J}_{m+2} \mathbb{J}_{n+1}-\mathbb{J}_{m+1} \mathbb{J}_{n+2}=(-1)^{n+2} 2^{n+1} J_{m-n}\left(p^{2}+p q-2 q^{2}\right),
$$




$$
\mathbb{J}_{m+2} \mathbb{J}_{n}-\mathbb{J}_{m} \mathbb{J}_{n+2}=(-1)^{n+1} 2^{n} J_{m-n}\left(p^{2}+p q-2 q^{2}\right)
$$

and

$$
\mathbb{J}_{m+1} \mathbb{J}_{n}-\mathbb{J}_{m} \mathbb{J}_{n+1}=(-1)^{n+1} 2^{n} J_{m-n}\left(p^{2}+p q-2 q^{2}\right) .
$$

Then from the equations (40), (41) and (42), we obtain the equation (38).

Theorem 5. Let $\overrightarrow{\mathbb{J}_{\mathrm{n}}}, \overrightarrow{\mathbb{J}_{\mathrm{m}}}$ and $\overrightarrow{\mathbb{J}_{l}}$ be the generalized Jacobsthal vectors. The mixed product of these vectors is

$$
\left\langle\overrightarrow{\mathbb{J}_{\mathrm{n}}} \times \overrightarrow{\mathbb{J}_{\mathrm{m}}}, \overrightarrow{\mathbb{J}_{l}}\right\rangle=0
$$

Proof. Using $\overrightarrow{\mathbb{J}_{l}}=\left(\mathbb{J}_{l}, \mathbb{J}_{l+1}, \mathbb{J}_{l+2}\right)$, we can write,

$$
\begin{array}{r}
\left\langle\overrightarrow{\mathbb{J}_{\mathrm{n}}} \times \overrightarrow{\mathbb{J}_{\mathrm{m}}}, \quad \overrightarrow{\mathbb{J}_{l}}\right\rangle=\left|\begin{array}{ccc}
\mathbb{J}_{n} & \mathbb{J}_{n+1} & \mathbb{J}_{n+2} \\
\mathbb{J}_{m} & \mathbb{J}_{m+1} & \mathbb{J}_{m+2} \\
\mathbb{J}_{l} & \mathbb{J}_{l+1} & \mathbb{J}_{l+2}
\end{array}\right|=\mathbb{J}_{n}\left(\mathbb{J}_{m+1} \mathbb{J}_{l+2}-\mathbb{J}_{m+2} \mathbb{J}_{l+1}\right) \\
+\mathbb{J}_{n+1}\left(\mathbb{J}_{m+2} \mathbb{J}_{l}-\mathbb{J}_{m} \mathbb{J}_{l+2}\right)+\mathbb{J}_{n+2}\left(\mathbb{J}_{m} \mathbb{J}_{l+1}-\mathbb{J}_{m+1} \mathbb{J}_{l}\right) .
\end{array}
$$

Also, using the equations (40), (41) and (42) we obtain

$$
\begin{aligned}
\mathbb{J}_{n}\left(\mathbb{J}_{m+1} \mathbb{J}_{l+2}-\mathbb{J}_{m+2} \mathbb{J}_{l+1}\right)+\mathbb{J}_{n+1}\left(\mathbb{J}_{m+2} \mathbb{J}_{l}-\mathbb{J}_{m} \mathbb{J}_{l+2}\right) & +\mathbb{J}_{n+2}\left(\mathbb{J}_{m} \mathbb{J}_{l+1}-\mathbb{J}_{l} \mathbb{J}_{m+1}\right) \\
= & (-1)^{l} 2^{l} J_{m-l}\left(p^{2}+p q-2 q^{2}\right) \\
& \left(-2 \mathbb{J}_{n}-\mathbb{J}_{n+1}+\mathbb{J}_{n+2}\right) \\
= & (-1)^{l} 2^{l} J_{m-l} e_{J}\left(-\mathbb{J}_{n+2}+\mathbb{J}_{n+2}\right)=0 .
\end{aligned}
$$

Thus, we have the equation (43).

\section{The generalized complex Jacobsthal sequence}

In this section, the generalized complex Jacobsthal sequence, denoted by $\mathbb{C}_{n}$, will be defined. The generalized complex Jacobsthal sequence is defined by

$$
\mathbb{C}_{n}=\mathbb{J}_{n}+i \mathbb{J}_{n+1},
$$

with $\mathbb{C}_{0}=q+i(p+q), \mathbb{C}_{1}=(p+q)+i(p+3 q), \mathbb{C}_{2}=(p+3 q)+i(3 p+5 q)$, where $p, q$ are arbitrary integers. That is, the generalized complex Jacobsthal sequence is

$$
\begin{array}{r}
q+i(p+q),(p+q)+i(p+3 q),(p+3 q)+i(3 p+5 q), \\
(3 p+5 q)+i(5 p+11 q), \ldots,(p+i 2 q) J_{n}+(q+i(p+q)) J_{n+1}, \ldots
\end{array}
$$

- Special case 1: From the generalized complex Jacobsthal sequence $\left(\mathbb{C}_{n}\right)$ for $p=1, q=0$ in the equation (47), we obtain complex Jacobsthal sequence $\left(C_{n}=J_{1, i}\right)$ as follows:

$$
\left(C_{n}\right): i, 1+i, 1+i 3,3+i 5,5+i 11, \ldots, J_{n}+i J_{n+1}, \ldots
$$


- Special case 2: From the generalized complex Jacobsthal sequence $\left(\mathbb{C}_{n}\right)$ for $p=-1, q=$ 2 in the equation (47), we obtain complex Jacobsthal-Lucas sequence $\left(C_{n}=j_{-1+4 i, 2+i}\right)$ as follows:

$$
2+i, 1+i 5,5+i 7,7+i 17,17+i 31, \ldots, j_{n}+i j_{n+1}, \ldots
$$

For the generalized complex Jacobsthal sequence, we have the following properties:

$$
\begin{gathered}
\mathbb{C}_{n}^{2}+2 \mathbb{C}_{n-1}^{2}=[(2 p+q)+i(p+5 q)] \mathbb{C}_{2 n-1}-(3+i) e_{J} J_{2 n-1} \\
\mathbb{C}_{n+1}^{2}+2 \mathbb{C}_{n}^{2}=[(2 p+q)+i(p+5 q)] \mathbb{C}_{2 n+1}-(3+i) e_{J} J_{2 n+1} \\
\mathbb{C}_{n+1}^{2}-4 \mathbb{C}_{n-1}^{2}=[(2 p+q)+i(p+5 q)] \mathbb{C}_{2 n}-(3+i) e_{J} J_{2 n} \\
\mathbb{C}_{n-1} \mathbb{C}_{n+1}-\mathbb{C}_{n}^{2}=(-1)^{n} 2^{n-1}(3+i) e_{J},
\end{gathered}
$$

where $e_{J}=p^{2}+p q-2 q^{2}$.

\section{The generalized dual Jacobsthal sequence}

In this section, the generalized dual Jacobsthal sequence, denoted by $\mathbb{D}_{n}^{J}$, will be defined. The generalized dual Jacobsthal sequence is defined by

$$
\mathbb{D}_{n}^{J}=\mathbb{J}_{n}+\varepsilon \mathbb{J}_{n+1},
$$

with $\mathbb{D}_{0}^{J}=q+\varepsilon(p+q), \mathbb{D}_{1}^{J}=p+q+\varepsilon(p+3 q), \mathbb{D}_{2}^{J}=p+3 q+\varepsilon(3 p+5 q)$, where $p, q$ are arbitrary integers. That is, the generalized dual Jacobsthal sequence is

$$
\begin{gathered}
q+\varepsilon(p+q),(p+q)+\varepsilon(p+3 q),(p+3 q)+\varepsilon(3 p+5 q), \\
(3 p+5 q)+\varepsilon(5 p+11 q),(5 p+11 q)+\varepsilon(11 p+21 q), \\
\ldots,\left[(p+2 \varepsilon q) J_{n}+(q+\varepsilon(p+q)) J_{n+1}=\mathbb{J}_{n}+\varepsilon \mathbb{J}_{n+1}, \ldots\right.
\end{gathered}
$$

Using the equations (52) and (53), we get

$$
\begin{aligned}
& \mathbb{D}_{n}^{J}=(p+2 \varepsilon q) J_{n}+(q+\varepsilon(p+q)) J_{n+1} \\
& \mathbb{D}_{n+1}^{J}=2\left[(q+\varepsilon(p+q)] J_{n}+[(p+q)+\varepsilon(p+3 q)] J_{n+1}\right. \\
& \mathbb{D}_{n+2}^{J}=2[(p+q)+\varepsilon(p+3 q)] J_{n}+[(p+3 q)+\varepsilon(3 p+5 q)] J_{n+1} \\
& \vdots \\
& \mathbb{D}_{n+r}^{J}=2 \mathbb{D}_{n-1}^{J} J_{r}+\mathbb{D}_{n}^{J} J_{r+1}
\end{aligned}
$$

- Special case 1: From the generalized dual Jacobsthal sequence $\left(\mathbb{D}_{n}^{J}\right)$ for $p=1, q=0$ in the equation (53), we obtain dual Jacobsthal sequence $\left(D_{n}^{J}\right)$ as follows:

$$
\left(D_{n}^{J}\right): \varepsilon, 1+\varepsilon, 1+3 \varepsilon, 3+5 \varepsilon, 5+11 \varepsilon, \ldots, J_{n}+\varepsilon J_{n+1}, \ldots
$$

- Special case 2: From the generalized dual Jacobsthal sequence $\left(\mathbb{D}_{n}^{J}\right)$ for $p=-1, q=2$ in the equation (53), we obtain dual Jacobsthal-Lucas sequence $\left(D_{n}^{j}\right)$ as follows:

$$
\left(D_{n}^{j}\right): 2+\varepsilon, 1+5 \varepsilon, 5+7 \varepsilon, 7+17 \varepsilon, 17+31 \varepsilon, \ldots, j_{n}+\varepsilon j_{n+1}, \ldots
$$


For the generalized dual Jacobsthal sequence, we have the following properties:

$$
\begin{gathered}
\left(\mathbb{D}_{n}^{J}\right)^{2}+2\left(\mathbb{D}_{n}^{J}\right)^{2}=[(2 p+q)+\varepsilon(p+5 q)] \mathbb{D}_{2 n-1}^{J}-(1+\varepsilon) e_{J} J_{2 n-1}, \\
\left(\mathbb{D}_{n+1}^{J}\right)^{2}+2\left(\mathbb{D}_{n}^{J}\right)^{2}=[(2 p+q)+\varepsilon(p+5 q)] \mathbb{D}_{2 n+1}^{J}-(1+\varepsilon) e_{J} J_{2 n+1}, \\
\left(\mathbb{D}_{n+1}^{J}\right)^{2}-4\left(\mathbb{D}_{n-1}^{J}\right)^{2}=[(2 p+q)+\varepsilon(p+5 q)] \mathbb{D}_{2 n}^{J}-(1+\varepsilon) e_{J} J_{2 n}, \\
\mathbb{D}_{n-1}^{J} \mathbb{D}_{n+1}^{J}-\left(\mathbb{D}_{n}^{J}\right)^{2}=(-1)^{n} 2^{n-1}(1+\varepsilon) e_{J} \\
4\left(\mathbb{D}_{n}^{J}\right)^{2}+2 J_{n+1}^{2}(1+\varepsilon) e_{J}=2(p+2 \varepsilon q) \mathbb{D}_{2 n+1}^{J}, \\
\mathbb{D}_{m}^{J} \mathbb{D}_{n+1}^{J}+2 \mathbb{D}_{m-1}^{J} \mathbb{D}_{n}^{J}=(2 p+q)+\varepsilon(p+5 q) \mathbb{D}_{m+n}^{J}-(1+\varepsilon) e_{J} J_{m+n}, \\
\mathbb{D}_{m}^{J} \mathbb{D}_{n-1}^{J}-\mathbb{D}_{m-1}^{J} \mathbb{D}_{n}^{J}=(-1)^{n} 2^{n-1}(1+\varepsilon) e_{J} J_{m-n} .
\end{gathered}
$$

where $e_{J}=\left(p^{2}+p q-2 q^{2}\right)$.

- Special case 3: From properties of the generalized dual Jacobsthal sequence $\left(\mathbb{D}_{n}^{J}\right)$ for $p=1, \quad q=0$ in the equations (55)-(61), we obtain dual Jacobsthal sequence $\left(D_{n}^{J}\right)$ as follows:

$$
\begin{gathered}
\left(D_{n}^{J}\right)^{2}+2\left(D_{n-1}^{J}\right)^{2}=(2+\varepsilon) D_{2 n-1}^{J}-(1+\varepsilon) J_{2 n-1}, \\
\left(D_{n+1}^{J}\right)^{2}+2\left(D_{n}^{J}\right)^{2}=(2+\varepsilon) D_{2 n+1}^{J}-(1+\varepsilon) J_{2 n+1}, \\
\left(D_{n+1}^{J}\right)^{2}-4\left(D_{n-1}^{J}\right)^{2}=(2+\varepsilon) D_{2 n}^{J}-(1+\varepsilon) J_{2 n} \\
D_{n-1}^{J} D_{n+1}^{J}-\left(D_{n}^{J}\right)^{2}=(-1)^{n} 2^{n-1}(1+\varepsilon), \\
4\left(D_{n}^{J}\right)^{2}+2(1+\varepsilon) J_{n+1}^{2}=2 D_{2 n+1}^{J}, \\
D_{m}^{J} D_{n+1}^{J}+2 D_{m-1}^{J} D_{n}^{J}=(2+\varepsilon) D_{m+n}^{J}-(1+\varepsilon) J_{m+n}, \\
D_{m}^{J} D_{n-1}^{J}-D_{m-1}^{J} D_{n}^{J}=(-1)^{n} 2^{n-1}(1+\varepsilon) J_{m-n} .
\end{gathered}
$$

Theorem 6. If $\mathbb{D}_{n}^{J}$ is the generalized dual Jacobsthal number, then

$$
\lim _{n \rightarrow \infty} \frac{\mathbb{D}_{n+1}^{J}}{\mathbb{D}_{n}^{J}}=\frac{\left(p q+q^{2}\right) \alpha^{2}+\left(p^{2}+p q+2 q^{2}\right) \alpha+2 p q}{q^{2} \alpha^{2}+(2 p q) \alpha+p^{2}}
$$

where $\alpha=-1$.

Proof. For the generalized dual Jacobsthal number $\mathbb{D}_{n}^{J}$, we obtain

$$
\begin{aligned}
\lim _{n \rightarrow \infty} & \frac{\mathbb{D}_{n+1}^{J}}{\mathbb{D}_{n}^{J}}=\lim _{n \rightarrow \infty} \frac{(p+2 \varepsilon q) J_{n+1}+(q+\varepsilon(p+q)) J_{n+2}}{(p+2 \varepsilon q) J_{n}+[q+\varepsilon(p+q)] J_{n+1}} \\
= & \lim _{n \rightarrow \infty} \frac{\left(p^{2}+p q+2 q^{2}\right) J_{n} J_{n+1}+\left(p q+q^{2}\right)\left(J_{n+1}^{2}+2 p q J_{n}^{2}\right)}{p^{2} J_{n}^{2}+2 p q J_{n} J_{n+1}+q^{2} J_{n+1}^{2}} \\
& \quad+\lim _{n \rightarrow \infty} \varepsilon \frac{(-1)^{n+1} 2^{n}\left(p^{2}+p q-2 q^{2}\right)}{p^{2} J_{n}^{2}+2 p q J_{n} J_{n+1}+q^{2} J_{n+1}^{2}} \\
= & \frac{\left(p^{2}+p q+2 q^{2}\right) \alpha+\left(p q+q^{2}\right) \alpha^{2}+(2 p q)}{q^{2} \alpha^{2}+(2 p q) \alpha+p^{2}}
\end{aligned}
$$

where $J_{n+2}=J_{n+1}+2 J_{n}$. 
- Special case 4: For $p=1, q=0$ in the equation (69), we obtain

$$
\lim _{n \rightarrow \infty} \frac{\mathbb{D}_{n+1}^{J}}{\mathbb{D}_{n}^{J}}=\lim _{n \rightarrow \infty} \frac{D_{n+1}^{J}}{D_{n}^{J}}=\alpha+0=\alpha .
$$

Theorem 7. The Binet's formula for the generalized dual Jacobsthal sequence is as follows;

$$
\mathbb{D}_{n}^{J}=\frac{1}{\alpha-\beta}\left(\bar{\alpha} \alpha^{n}-\bar{\beta} \beta^{n}\right) .
$$

Proof. If we use definition of the generalized dual Jacobsthal sequence and substitute first equation in footnote, then we get

$$
\begin{aligned}
\mathbb{D}_{n}^{J} & =(p+2 \varepsilon q) J_{n}+(q+\varepsilon(p+q)) J_{n+1} \\
& =(p+2 \varepsilon q)\left(\frac{\alpha^{n}-\beta^{n}}{\alpha-\beta}\right)+(q+\varepsilon(p+q))\left(\frac{\alpha^{n+1}-\beta^{n+1}}{\alpha-\beta}\right) \\
& =\frac{\bar{\alpha} \alpha^{n}-\bar{\beta} \beta^{n}}{\alpha-\beta}
\end{aligned}
$$

where $\bar{\alpha}=(p+2 \varepsilon q)+\alpha(q+\varepsilon(p+q))$ and $\bar{\beta}=(p+2 \varepsilon q)+\beta(q+\varepsilon(p+q))$.

\section{The generalized dual Jacobsthal vectors}

A generalized dual Jacobsthal vector is defined by

$$
\overrightarrow{\mathbb{D}}_{n}^{J}=\left(\mathbb{D}_{n}^{J}, \mathbb{D}_{n+1}^{J}, \mathbb{D}_{n+2}^{J}\right) .
$$

From the equations (52), (53) and (54) it can be expressed as

$$
\overrightarrow{\mathbb{D}}_{n}^{J}=\overrightarrow{\mathbb{J}}_{n}+\varepsilon \overrightarrow{\mathbb{J}}_{n+1}=(p+2 \varepsilon q) \vec{J}_{n}+(q+\varepsilon(p+q)) \vec{J}_{n+1}
$$

where $\overrightarrow{\mathbb{J}}_{n}=\left(\mathbb{J}_{n}, \mathbb{J}_{n+1}, \mathbb{J}_{n+2}\right)$ and $\vec{J}_{\mathrm{n}}=\left(J_{n}, J_{n+1}, J_{n+2}\right)$ are the generalized Jacobsthal vector and the Jacobsthal vector, respectively. The product of $\overrightarrow{\mathbb{D}}_{n}$ and $\lambda \in \mathbb{R}$ is given by

$$
\lambda \overrightarrow{\mathbb{D}}_{n}^{J}=\lambda \overrightarrow{\mathbb{J}}_{n}+\varepsilon \lambda \overrightarrow{\mathbb{J}}_{n+1}
$$

and $\overrightarrow{\mathbb{D}}_{n}^{j}$ and $\overrightarrow{\mathbb{D}}_{m}^{j}$ are equal if and only if

$$
\mathbb{J}_{\mathrm{n}}=\mathbb{J}_{\mathrm{m}}, \mathbb{J}_{\mathrm{n}+1}=\mathbb{J}_{\mathrm{m}+1}, \mathbb{J}_{\mathrm{n}+2}=\mathbb{J}_{\mathrm{m}+2} \text {. }
$$

Some examples of the generalized dual Jacobsthal vectors can be given easily as:

$$
\begin{aligned}
{\overrightarrow{\mathbb{D}^{J}}}_{1} & =\left(\mathbb{D}_{1}^{J}, \mathbb{D}_{2}^{J}, \mathbb{D}_{3}^{J}\right)=\left(\mathbb{J}_{1}, \mathbb{J}_{2}, \mathbb{J}_{3}\right)+\varepsilon\left(\mathbb{J}_{2}, \mathbb{J}_{3}, \mathbb{J}_{4}\right) \\
& =[(p+q)+\varepsilon(p+3 q),(p+3 q)+\varepsilon(3 p+5 q),(3 p+5 q)+\varepsilon(5 p+11 q)], \\
{\overrightarrow{\mathbb{D}^{J}}}_{2} & =\left(\mathbb{D}_{2}^{J}, \mathbb{D}_{3}^{J}, \mathbb{D}_{4}^{J}\right)+\varepsilon\left(\mathbb{D}_{3}^{J}, \mathbb{D}_{4}^{J}, \mathbb{D}_{5}^{J}\right) \\
& =[(p+3 q)+\varepsilon(3 p+5 q),(3 p+5 q)+\varepsilon(5 p+11 q),(5 p+11 q)+\varepsilon(11 p+21 q)] .
\end{aligned}
$$


Theorem 8. Let $\overrightarrow{\mathbb{D}}_{n}^{j}$ and $\overrightarrow{\mathbb{D}}_{m}^{j}$ be two generalized dual Jacobsthal vectors. The dot product of $\overrightarrow{\mathbb{D}}_{n}^{J}$ and $\overrightarrow{\mathbb{D}}_{m}^{J}$ is given by

$$
\begin{aligned}
\left\langle\overrightarrow{\mathbb{D}}_{n}^{j}, \overrightarrow{\mathbb{D}}_{m}^{j}\right\rangle= & p^{2}\left[\frac{1}{3}\left(J_{n+m}+J_{n+m+2}+J_{n+m+4}\right)\right. \\
& \left.+(-1)^{n+1} J_{m}+(-1)^{m+1} J_{n}\right] \\
& +2 p q\left[\frac{1}{3}\left(J_{n+m+1}+J_{n+m+3}+J_{n+m+5}\right)\right. \\
& \left.+(-1)^{n+1} J_{m-1}+(-1)^{m+1} J_{n-1}\right] \\
& +q^{2}\left[\frac{1}{3}\left(J_{n+m+2}+J_{n+m+4}+J_{n+m+6}\right)\right. \\
& \left.+(-1)^{n+2} J_{m+1}+(-1)^{m+2} J_{n+1}\right] \\
& +2 \varepsilon\left\{p ^ { 2 } \left[\frac{1}{3}\left(J_{n+m+1}+J_{n+m+3}+J_{n+m+5}\right)\right.\right. \\
& \left.+(-1)^{n+1} J_{m-1}+(-1)^{m+1} J_{n-1}\right] \\
& +2 p q\left[\frac{1}{3}\left(J_{n+m+2}+J_{n+m+4}+J_{n+m+6}\right)\right. \\
& \left.+(-1)^{n+1} J_{m-2}+(-1)^{m+1} J_{n-2}\right] \\
& +q^{2}\left[\frac{1}{3}\left(J_{n+m+3}+J_{n+m+5}+J_{n+m+7}\right)\right. \\
& \left.\left.+(-1)^{n+2} J_{m}+(-1)^{m+2} J_{n}\right]\right\} .
\end{aligned}
$$

Proof. The dot product of $\overrightarrow{\mathbb{D}}_{n}^{J}=\left(\mathbb{D}_{n}^{J}, \mathbb{D}_{n+1}^{J}, \mathbb{D}_{n+2}^{J}\right)$ and $\overrightarrow{\mathbb{D}}_{m}^{J}=\left(\mathbb{D}_{m}^{J}, \mathbb{D}_{m+1}^{J}, \mathbb{D}_{m+2}^{J}\right)$ is defined by

$$
\begin{aligned}
\left\langle\overrightarrow{\mathbb{D}}_{n}^{J}, \overrightarrow{\mathbb{D}}_{m}^{J}\right\rangle & =\mathbb{D}_{n}^{J} \mathbb{D}_{m}^{J}+\mathbb{D}_{n+1}^{J} \mathbb{D}_{m+1}^{J}+\mathbb{D}_{n+2}^{J} \mathbb{D}_{m+2}^{J} \\
& =\left\langle\overrightarrow{\mathbb{J}}_{n}, \overrightarrow{\mathbb{J}}_{m}\right\rangle+\varepsilon\left[\left\langle\overrightarrow{\mathbb{J}}_{n}, \overrightarrow{\mathbb{J}}_{m+1}\right\rangle+\left\langle\overrightarrow{\mathbb{J}}_{n+1}, \overrightarrow{\mathbb{J}}_{m}\right\rangle\right],
\end{aligned}
$$

where $\overrightarrow{\mathbb{J}}_{n}=\left(\mathbb{J}_{n}, \mathbb{J}_{n+1}, \mathbb{J}_{n+2}\right)$ is the generalized Jacobsthal vector. Also, the equations (11), (12) and (13), we obtain

$$
\begin{aligned}
\left\langle\overrightarrow{\mathbb{J}}_{n}, \overrightarrow{\mathbb{J}}_{m}\right\rangle= & p^{2}\left[\frac{1}{3}\left(J_{n+m}+J_{n+m+2}+J_{n+m+4}\right)\right. \\
& \left.+(-1)^{n+1} J_{m}+(-1)^{m+1} J_{n}\right] \\
& +2 p q\left[\frac{1}{3}\left(J_{n+m+1}+J_{n+m+3}+J_{n+m+5}\right)\right. \\
& \left.+(-1)^{n+1} J_{m-1}+(-1)^{m+1} J_{n-1}\right] \\
& +q^{2}\left[\frac{1}{3}\left(J_{n+m+2}+J_{n+m+4}+J_{n+m+6}\right)\right. \\
& \left.+(-1)^{n+2} J_{m+1}+(-1)^{m+2} J_{n+1}\right]
\end{aligned}
$$




$$
\begin{aligned}
\left\langle\overrightarrow{\mathbb{J}}_{n}, \overrightarrow{\mathbb{J}}_{m+1}\right\rangle= & p^{2}\left[\frac{1}{3}\left(J_{n+m+1}+J_{n+m+3}+J_{n+m+5}\right)\right. \\
& \left.+(-1)^{n+1} J_{m+1}+(-1)^{m+2} J_{n}\right] \\
& +2 p q\left[\frac{1}{3}\left(J_{n+m+2}+J_{n+m+4}+J_{n+m+6}\right)\right. \\
& \left.+(-1)^{n+1} J_{m}+(-1)^{m+2} J_{n-1}\right] \\
& +q^{2}\left[\frac{1}{3}\left(J_{n+m+3}+J_{n+m+5}+J_{n+m+7}\right)\right. \\
& \left.+(-1)^{n+2} J_{m+2}+(-1)^{m+3} J_{n+1}\right], \\
\left\langle\overrightarrow{\mathbb{J}}_{n+1}, \overrightarrow{\mathbb{J}}_{m}\right\rangle= & p^{2}\left[\frac{1}{3}\left(J_{n+m+1}+J_{n+m+3}+J_{n+m+5}\right)\right. \\
& \left.+(-1)^{n+2} J_{m}+(-1)^{m+1} J_{n+1}\right] \\
& +2 p q\left[\frac{1}{3}\left(J_{n+m+2}+J_{n+m+4}+J_{n+m+6}\right)\right. \\
& \left.+(-1)^{n+2} J_{m-1}+(-1)^{m+1} J_{n}\right] \\
& +q^{2}\left[\frac{1}{3}\left(J_{n+m+3}+J_{n+m+5}+J_{n+m+7}\right)\right. \\
& \left.+(-1)^{n+3} J_{m+1}+(-1)^{m+2} J_{n+2}\right] .
\end{aligned}
$$

Then from equation (74), (75) and (76), we have the equation (73).

- Special case 1: For the dot product of generalized dual Jacobsthal vectors $\overrightarrow{\mathbb{D}}_{n}{ }_{n}$ and $\overrightarrow{\mathbb{D}}_{n+1}^{J}$, we get

$$
\begin{aligned}
\left\langle\overrightarrow{\mathbb{D}}_{n}^{J}, \overrightarrow{\mathbb{D}}_{n+1}^{J}\right\rangle= & \mathbb{D}_{n}^{J} \mathbb{D}_{n+1}^{J}+\mathbb{D}_{n+1}^{J} \mathbb{D}_{n+2}^{J}+\mathbb{D}_{n+2}^{J} \mathbb{D}_{n+3}^{J} \\
= & \left\langle\overrightarrow{\mathbb{J}}_{n}, \overrightarrow{\mathbb{J}}_{n+1}\right\rangle+\varepsilon\left\{\left\langle\overrightarrow{\mathbb{J}}_{n}, \overrightarrow{\mathbb{J}}_{n+2}\right\rangle+\left\langle\overrightarrow{\mathbb{J}}_{n+1}, \overrightarrow{\mathbb{J}}_{n+1}\right\rangle\right\} \\
= & \left\{p^{2}\left[\frac{1}{3}\left(J_{2 n+1}+J_{2 n+3}+J_{2 n+5}\right)+2(-1)^{n+1} J_{n-1}\right]\right. \\
& +2 p q\left[\frac{2}{3}\left(J_{2 n+2}+J_{2 n+4}+J_{2 n+6}\right)+2(-1)^{n+1} J_{n-2}\right] \\
& \left.+q^{2}\left[\frac{1}{3}\left(J_{2 n+3}+J_{2 n+5}+J_{2 n+7}\right)+2(-1)^{n+2} J_{n}\right]\right\} \\
+ & 2 \varepsilon\left\{p^{2}\left[\frac{1}{3}\left(J_{2 n+2}+J_{2 n+4}+J_{2 n+6}\right)+2(-1)^{n+1} J_{n-2}\right]\right. \\
& +p q\left[\frac{2}{3}\left(J_{2 n+3}+J_{2 n+5}+J_{2 n+7}\right)+4(-1)^{n+1} J_{n-3}\right] \\
& \left.+q^{2}\left[\frac{1}{3}\left(J_{2 n+4}+J_{2 n+6}+J_{2 n+8}\right)+2(-1)^{n+2} J_{n-1}\right]\right\} .
\end{aligned}
$$


Then for the norm of the generalized dual Jacobsthal vector ${ }^{3}$, we have

$$
\begin{aligned}
\left\|\overrightarrow{\mathbb{D}}_{n}{ }_{n}\right\|= & \sqrt{\left[\left\langle{\overrightarrow{\mathbb{D}^{J}}}_{n},{\overrightarrow{\mathbb{D}^{J}}}_{n}\right\rangle\right]}=\sqrt{\left[\left(\mathbb{D}_{n}^{J}\right)^{2}+\left(\mathbb{D}_{n+1}^{J}\right)^{2}+\left(\mathbb{D}_{n+2}^{J}\right)^{2}\right]} \\
= & \sqrt{\left[p^{2}\left(J_{2 n+3}+J_{n}^{2}-J_{n+1}^{2}\right)\right]} \\
& +\sqrt{2 p q\left[\left(\frac{1}{3}\left(J_{2 n+1}+J_{2 n+3}+J_{2 n+5}\right)+2(-1)^{n+1} J_{n-1}\right)\right]} \\
& +\sqrt{q^{2}\left[\left(J_{2 n+5}+J_{n+1}^{2}-J_{n+2}^{2}\right)\right]} \\
& +\sqrt{\varepsilon\left\{2 p^{2}\left(\frac{1}{3}\left(J_{2 n+1}+J_{2 n+3}+J_{2 n+5}\right)+4(-1)^{n+1} J_{n-1}\right)\right\}} \\
& +\sqrt{\varepsilon\left\{4 p q\left[\frac{1}{3}\left(J_{2 n+2}+J_{2 n+4}+J_{2 n+6}\right)+4(-1)^{n+1} J_{n-2}\right]\right\}} \\
& +\sqrt{\left.\varepsilon\left\{2 q^{2}\left(\frac{1}{3}\left(J_{2 n+3}+J_{2 n+5}+J_{2 n+7}\right)+4(-1)^{n+2} J_{n}\right)\right]\right\}}
\end{aligned}
$$

where used identity of the Jacobsthal numbers as follows [6]

$$
\begin{aligned}
J_{n} J_{n+k}= & \frac{1}{3}\left(J_{2 n+k}+(-1)^{n+1} J_{n+k}+(-1)^{n+k+1} J_{n} .\right. \\
\left\langle\overrightarrow{\mathbb{D}}_{n}^{J}, \overrightarrow{\mathbb{D}}_{n}^{J}\right\rangle= & \left(\mathbb{D}_{n}^{J}\right)^{2}+\left(\mathbb{D}_{n+1}^{J}\right)^{2}+\left(\mathbb{D}_{n+2}^{J}\right)^{2} \\
= & \left\langle\overrightarrow{\mathbb{J}}_{n}, \overrightarrow{\mathbb{J}}_{n}\right\rangle+2 \varepsilon\left\langle\overrightarrow{\mathbb{J}}_{n}, \overrightarrow{\mathbb{J}}_{n+1}\right\rangle \\
= & p^{2}\left[J_{2 n+3}+J_{n}^{2}-J_{n+1}^{2}\right] \\
& +2 p q\left[\frac{1}{3}\left(J_{2 n+1}+J_{2 n+3}+J_{2 n+5}\right)\right. \\
& \left.+2(-1)^{n+1} J_{n-1}\right]+q^{2}\left[J_{2 n+5}+J_{n+1}^{2}-J_{n+2}^{2}\right] \\
& +\varepsilon\left\{2 p^{2}\left[\frac{1}{3}\left(J_{2 n+1}+J_{2 n+3}+J_{2 n+5}\right)+2(-1)^{n+1} J_{n-1}\right]\right. \\
& +4 p q\left[\frac{1}{3}\left(J_{2 n+2}+J_{2 n+4}+J_{2 n+6}\right)+2(-1)^{n+1} J_{n-2}\right] \\
& \left.+2 q^{2}\left[\frac{1}{3}\left(J_{2 n+3}+J_{2 n+5}+J_{2 n+7}\right)+2(-1)^{n+2} J_{n}\right]\right\} .
\end{aligned}
$$

- Special case 2: For $p=1, q=0$, in the equations (73), (77) and (79), we have

$$
\begin{aligned}
& \left\langle\vec{D}_{n}^{J}, \vec{D}_{m}^{J}\right\rangle=\left[\frac{1}{3}\left(J_{n+m}+J_{n+m+2}+J_{n+m+4}\right)+(-1)^{n+1} J_{m}+(-1)^{m+1} J_{n}\right] \\
& +2 \varepsilon\left[\frac{1}{3}\left(J_{n+m+1}+J_{n+m+3}+J_{n+m+5}\right)+(-1)^{n+1} J_{m-1}+(-1)^{m+1} J_{n-1}\right], \\
& \left\langle\vec{D}_{n}^{J}, \vec{D}_{n+1}\right\rangle=\left[\frac{1}{3}\left(J_{2 n+1}+J_{2 n+3}+J_{2 n+5}\right)+2(-1)^{n+1} J_{n-1}\right] \\
& +2 \varepsilon\left[\frac{1}{3}\left(J_{2 n+2}+J_{2 n+4}+J_{2 n+6}\right)+2(-1)^{n+1} J_{n-2}\right]
\end{aligned}
$$

${ }^{3}$ Norm of dual number as follows: $\|\overrightarrow{\mathbb{A}}\|=\sqrt{a+\varepsilon a^{*}}=\sqrt{a}+\varepsilon a^{*} \frac{1}{2 \sqrt{a}}, A=a+\varepsilon a^{*},[1]$. 
and

$$
\begin{aligned}
& \left\|\vec{D}_{n}^{j}\right\|=\sqrt{\left(J_{2 n+3}+J_{n}^{2}-J_{n+1}^{2}\right)} \\
& +\sqrt{2 \varepsilon\left[\frac{1}{3}\left(J_{2 n+1}+J_{2 n+3}+J_{2 n+5}\right)+2(-1)^{n+1} J_{n-1}\right]} \\
& =\left(J_{2 n+3}+J_{n}^{2}-J_{n+1}^{2}\right)+\varepsilon \frac{\frac{1}{3}\left[\left(J_{2 n+1}+J_{2 n+3}+J_{2 n+5}\right)+2(-1)^{n+1} J_{n-1}\right]}{\sqrt{\left(J_{2 n+3}+J_{n}^{2}-J_{n+1}^{2}\right)}} .
\end{aligned}
$$

Theorem 9. Let $\overrightarrow{\mathbb{D}}_{n}^{J}$ and $\overrightarrow{\mathbb{D}}_{m}^{J}$ be two generalized dual Jacobsthal vectors. The cross product of $\overrightarrow{\mathbb{D}^{J}}$ and $\overrightarrow{\mathbb{D}^{J}}{ }_{m}$ is given by

$$
{\overrightarrow{\mathbb{D}^{J}}}_{n} \times{\overrightarrow{\mathbb{D}^{j}}}_{m}=(-1)^{n+1} 2^{n} J_{m-n}(1+\varepsilon)\left(p^{2}+p q-2 q^{2}\right)(-2 i-j+k) .
$$

Proof. The cross product of $\overrightarrow{\mathbb{D}}_{n}^{j}=\overrightarrow{\mathbb{J}}_{n}+\varepsilon \overrightarrow{\mathbb{J}}_{n+1}$ and ${\overrightarrow{\mathbb{D}^{j}}}_{m}=\overrightarrow{\mathbb{J}}_{m}+\varepsilon \overrightarrow{\mathbb{J}}_{m+1}$ is defined by

$$
{\overrightarrow{\mathbb{D}^{J}}}_{n} \times \overrightarrow{\mathbb{D}}_{m}^{J}=\left(\overrightarrow{\mathbb{J}}_{n} \times \overrightarrow{\mathbb{J}}_{m}\right)+\varepsilon\left(\overrightarrow{\mathbb{J}}_{n} \times \overrightarrow{\mathbb{J}}_{m+1}+\overrightarrow{\mathbb{J}}_{n+1} \times \overrightarrow{\mathbb{J}}_{m}\right)
$$

where $\overrightarrow{\mathbb{J}}_{n}$ is the generalized Jacobsthal vector and $\overrightarrow{\mathbb{J}}_{n} \times \overrightarrow{\mathbb{J}}_{m}$ is the cross product for the generalized Jacobsthal vectors $\overrightarrow{\mathbb{J}}_{n}$ and $\overrightarrow{\mathbb{J}}_{m}$.

Now, we calculate the cross products $\overrightarrow{\mathbb{J}}_{n} \times \overrightarrow{\mathbb{J}}_{m}, \overrightarrow{\mathbb{J}}_{n} \times \overrightarrow{\mathbb{J}}_{m+1}$ and $\overrightarrow{\mathbb{J}}_{n+1} \times \overrightarrow{\mathbb{J}}_{m}$. Using the property $J_{m} J_{n-1}-J_{m-1} J_{n}=(-1)^{n} 2^{n-1} J_{m-n}$, we get

$$
\begin{aligned}
\overrightarrow{\mathbb{J}}_{n} \times \overrightarrow{\mathbb{J}}_{m} & =(-1)^{n+1} 2^{n} J_{m-n}(-2 i-j+k)\left(p^{2}+p q-2 q^{2}\right) \\
\overrightarrow{\mathbb{J}}_{n} \times \overrightarrow{\mathbb{J}}_{m+1} & =(-1)^{n+1} 2^{n} J_{m-n+1}(-2 i-j+k)\left(p^{2}+p q-2 q^{2}\right)
\end{aligned}
$$

and

$$
\overrightarrow{\mathbb{J}}_{n+1} \times \overrightarrow{\mathbb{J}}_{m}=(-1)^{n+2} 2^{n+1} J_{m-n-1}(-2 i-j+k)\left(p^{2}+p q-2 q^{2}\right) .
$$

Then from the equations (81), (82) and (83), we obtain the equation (80).

- Special case 3: For $p=1, q=0$ in the equations (80), we have

$$
{\overrightarrow{D^{J}}}_{n} \times{\overrightarrow{D^{J}}}_{m}=(-1)^{n+1} 2^{n} J_{m-n}(1+\varepsilon)(-2 i-j+k) \text {. }
$$

Theorem 10. Let $\overrightarrow{\mathbb{D}}_{n}^{j}, \overrightarrow{\mathbb{D}}_{m}^{j}$ and $\overrightarrow{\mathbb{D}}_{l}^{j}$ be the generalized dual Jacobsthal vectors. The mixed product of these vectors is

$$
\left\langle\overrightarrow{\mathbb{D}}_{n}^{j} \times{\overrightarrow{\mathbb{D}^{j}}}_{m}, \overrightarrow{\mathbb{D}}_{l}^{j}\right\rangle=0
$$

Proof. Using the properties

$$
\overrightarrow{\mathbb{D}}_{n}^{j} \times \overrightarrow{\mathbb{D}}_{m}^{j}=\left(\overrightarrow{\mathbb{J}}_{n} \times \overrightarrow{\mathbb{J}}_{m}\right)+\varepsilon\left(\overrightarrow{\mathbb{J}}_{n} \times \overrightarrow{\mathbb{J}}_{m+1}+\overrightarrow{\mathbb{J}}_{n+1} \times \overrightarrow{\mathbb{J}}_{m}\right)
$$

and ${\overrightarrow{\mathbb{D}^{J}}}_{l}=\overrightarrow{\mathbb{J}}_{l}+\varepsilon \overrightarrow{\mathbb{J}}_{l+1}$, we can write,

$$
\begin{aligned}
\left\langle\overrightarrow{\mathbb{D}}_{n}^{j} \times \overrightarrow{\mathbb{D}}_{m}^{j}, \overrightarrow{\mathbb{D}}_{l}^{j}\right\rangle= & \left\langle\overrightarrow{\mathbb{J}}_{n} \times \overrightarrow{\mathbb{J}}_{m}, \overrightarrow{\mathbb{J}}_{l}\right\rangle+\varepsilon\left[\left\langle\overrightarrow{\mathbb{J}}_{n} \times \overrightarrow{\mathbb{J}}_{m}, \overrightarrow{\mathbb{J}}_{l+1}\right\rangle\right. \\
& \left.+\left\langle\overrightarrow{\mathbb{J}}_{n} \times \overrightarrow{\mathbb{J}}_{m+1}, \overrightarrow{\mathbb{J}}_{l}\right\rangle+\left\langle\overrightarrow{\mathbb{J}}_{n+1} \times \overrightarrow{\mathbb{J}}_{m}, \overrightarrow{\mathbb{J}}_{l+1}\right\rangle\right] .
\end{aligned}
$$


Then from equations (81), (82) and (83), we obtain

$$
\begin{gathered}
\left\langle(-2 i-j+k), \overrightarrow{\mathbb{J}}_{l}\right\rangle=-2 \mathbb{J}_{l}-\mathbb{J}_{l+1}+\mathbb{J}_{l+2}=0, \\
\left\langle(-2 i-j+k), \overrightarrow{\mathbb{J}}_{l+1}\right\rangle=-2 \mathbb{J}_{l+1}-\mathbb{J}_{l+2}+\mathbb{J}_{l+3}=0 .
\end{gathered}
$$

Thus, we have the equation (84).

\section{Conclusions}

The generalized Jacobsthal, the generalized complex Jacobsthal and the generalized dual Jacobsthal sequences have been introduced and studied. The use of such special sequences has increased significantly in quantum mechanics, quantum physics, etc.

\section{Acknowledgements}

The author thanks the anonymous referee for his/her suggestions which helped for the improvement of the paper.

\section{References}

[1] Agrawal, O. M. P. (1987) Hamilton operators and dual-number-quaternions in spatial kinematics, Mechanism and Machine Theory, 22, 6, 569-575.

[2] Atanassov, K. (2011) Remark on Jacobsthal numbers, Part 2. Notes on Number Theory and Discrete Mathematics, 17, 2, 37-39.

[3] Atanassov, K. (2012) Short remarks on Jacobsthal numbers, Notes on Number Theory and Discrete Mathematics, 18, 2, 63-64.

[4] Čerin, Z. (2007) Formulae for sums of Jacobsthal-Lucas numbers, Int. Math. Forum, 2, 1969-1984.

[5] Čerin, Z. (2007) Sums of squares and products of Jacobsthal numbers, Journal of Integer Sequences, 10, Article 07.2.5.

[6] Daşdemir, A. (2012) On the Jacobsthal numbers by matrix method, Fen Derg, 7, 1, 69-76.

[7] Daşdemir, A. (2014) A study on the Jacobsthal and Jacobsthal-Lucas numbers by matrix method, DUFED Journal of Sciences, 3, 1, 13-18.

[8] Djordjevic, G. B. (2000) Derivative sequences of generalized Jacobsthal and JacobsthalLucas polynomials, Fibonacci Quarterly, 38, 4, 334-338. 
[9] Djordjevic, G. B. (2008) Generalized Jacobsthal polynomials, Fibonacci Quarterly, 38, 3, 239-243.

[10] Gupta, V. K., \& Panvar, Y. K. (2012) Common factors of generalized Fibonacci, Jacobsthal and Jacobsthal-Lucas numbers, International Journal of Applied Mathematical Research, $1,4,377-382$.

[11] Horadam, A. F. (1963) Complex Fibonacci numbers and Fibonacci quaternions, The American Mathematical Monthly, 70, 3, 289-291.

[12] Horadam, A. F. (1988) Jacobsthal and Pell curves, Fibonacci Quarterly, 26, 79-83.

[13] Horadam, A. F. (1996) Jacobsthal representation numbers, Fibonacci Quarterly, 34, 40-54.

[14] Horadam, A. F. (1997) Jacobsthal representation polynomials, Fibonacci Quarterly, 35, 137-148.

[15] Köken, F., \& Bozkurt, D. (2008) On the Jacobsthal numbers by matrix methods, Int. J. Contemp Math. Sciences, 3, 13, 605-614.

[16] Köken, F., \& Bozkurt, D. (2008) On the Jacobsthal-Lucas numbers by matrix methods, Int. J. Contemp Math. Sciences, 3, 13, 1629-1633.

[17] Sloane, N. J. A. (1973) A Handbook of Integer Sequences, Academic Press.

[18] Syznal-Liana, A., \& Wloch, I. (2016) A note on Jacobsthal quaternions, Advances in Applied Clifford Algebras, 26, 1, 441-447.

[19] Torunbalcı Aydın, F., \& Yüce, S. (2017) A new approach to Jacobsthal quaternions, Filomat, $31,18,5567-5579$. 